\title{
Bronchobiliary Fistula due to Hydatid Liver Cyst and Role of Bile Leakage Test during Surgical Treatment
}

\author{
Egemen Cicek ${ }^{1}$, Cemalettin Koc ${ }^{1}$, Tolga Sahin ${ }^{1}$, Cihan Gokler ${ }^{1}$, Aydin Aktas ${ }^{1}$, Cuneyt Kayaalp ${ }^{1}$, \\ Akimasa $\mathrm{Nakao}^{2}$
}

Corresponding author:

Egemen Cicek, MD

Inonu University Department of Surgery

44315, Malatya, Turkey

Phone: + 904223774001

Mobile phone: +905064940423

E-mail: dregemencicek@gmail.com

\section{Abbreviations:}

BBF - Bronchobiliary fistula;

AST - Aspartate amino transferase;

ALT - alanine amino transferase;

CT - computed tomography.
IInonu University Turgut Ozal Medical Center Institute of Liver Transplantation and Faculty of Medicine Department of Surgery, Malatya, Turkey

${ }^{2}$ Nagoya University Department of Gastroenterological Surgery

(surgery II) and President of JR Nagoya Central Hospital, Nagoya, Japan

\section{ABSTRACT}

Background: Bronchobiliary fistula (BBF) is a rare complication of the hydatid liver disease. The diagnosis depends on clinical suspicion and radiologic evaluation. Surgical treatment is the mainstay way for the complicated hydatid liver cysts. However, postoperative biliary fistula can make the situation more complicated. Bile leak test may be helpful for the prevention of this complication. In this study, the surgical management of a patient who developed BBF due to liver hydatid cyst was discussed with the literature.

Case Presentation: A patient (45-year-old female) withbilioptysis was diagnosed as BBF due to hydatid liver disease (segment VII, $7 \mathrm{~cm}$ ) and operated via transabdominal approach. Intraoperative cholangiography confirmed the BBF and hydatid liver cyst was treated by partial pericystectomy. Bile leakwas determined by leak testsusing methylene blue, biliary orifices were sutured and cavity was managed by omentoplasty. Furthermore, the fistula tract to the bronchi was closed by sutures. Postoperative course was uneventful and she was discharged on postoperative 7th day. After a 14 months follow-up, there was no recurrence and she was asymptomatic.

Conclusions: Bile leak test can be used in complicated hydatid liver cysts such as BBF. Key words: hepatic disease, echinococcus granulosus, diaphragmatic, pulmonary

\section{INTRODUCTION}

Hydatid cyst is a zoonotic disorder and frequently affects the liver. It is still endemic in major part of the world such as Mediterranean region, South America, Middle East, Asia and Africa and in the non-endemic regions it is encountered in the immigrants. The most frequent complication includes biliary involvement (1). In addition, rupture to the peritoneal cavity is the second most frequent complication of the disease $(2,3)$.

BBF can be defined as an abnormal connection between the biliary tract and the bronchial tree. Underlying etiology can be congenital or acquired. Among the acquired reasons are tumors, trauma, abscess, cholangitis due to choledo- 
cholithiasis, miscellaneous infective conditions of the liver. In endemic regions hydatid liver disease is the most frequent etiology of BBF. A systematic review of acquired BBF showed that hydatid liver disease constitutes $11.7 \%$ of the cases (4). BBF was first defined by Peacock 167 years ago. The patient that was initially defined to have BBF had underlying hydatid liver disease (5). The diagnosis is actually quite simple because bilioptysis is pathognomic for BBF (4). We did not find any study defining intraoperative bile leak test and bronchogram for the treatment of the hydatid liver disease. The aim of the present case report is to share the advantages of these diagnostic approaches in the treatment of a complicated hydatid liver disease; especially during the index procedure.

\section{CASE REPORT}

Forty-nine-year-old female patient with abdominal pain that had been present for 48 hours was admitted to our department. She had a productive cough with bilioptysis with a daily volume of 1000 cc sputum. She did not complain of any dyspnea. Her vital signs were normal (body temperature: $36.7^{\circ} \mathrm{C}$, blood pressure: 125/75 mm-Hg, pulse: 72 beats/min, respiratory rate: 22 respirations/min). Her laboratory values were as follows; leukocyte: 14.8 × $10^{3}$ cells $/ \mathrm{mm}^{3}$ (4.3-10.3), hemoglobin: 9.1g/dL (13.6-17.2), albumin: $3.6 \mathrm{~g} / \mathrm{dL}$ (3.4-4.8), Aspartate amino transferase (AST): $11 \mathrm{U} / \mathrm{L}$ (534), alanine amino transferase (ALT): $7 \mathrm{U} / \mathrm{L}(<55)$, gamma glutamyl transferase: $20 \mathrm{U} / \mathrm{L}(9-64)$, alkaline phosphatase: $97 \mathrm{U} / \mathrm{L}$ (40-150) and total bilirubin: 0.28 $\mathrm{mg} / \mathrm{dL}(0.2-1.2)$ direct bilirubin: $0.16 \mathrm{mg} / \mathrm{dL}(<0.5)$. The chest radiogram of the patient showed infiltration and atelectasis in the lower lobe of the right lung. Abdominal ultrasonography showed a $7 \mathrm{~cm}$ cystic lesion with air echo patterns and calcific foci in the junction of segment 7 and 8 of the liver. The biliary tree was observed to be normal and there were multiple millimetric calculi in the gallbladder. In the abdominal computed tomography (CT) there was a $7.5 \times 6 \mathrm{~cm}$ cystic mass which contained calcifications and free air and was accompanied with subcapsular $1 \mathrm{~cm}$ free fluid occupying segments 7-8. In the neighbouring anterolateral segment of the inferior lobe of the right lung, there were infiltration areas with air-bronchogram sign (fig. $1 a, b)$.

The CT images suggested ruptured hydatid disease of the liver. Together with the clinical characteristics of the patient and the imaging studies we decided to proceed with operative intervention with the diagnosis of complicated hydatid liver disease with BBF. A"J" incision was performed and a cyst occupying segment 7 and 8 that was adherent to the diaphragm was seen (fig. 2a). The intra-operative cholangiogram showed that cyst ruptured in to the biliary system and the bronchial tree of the right lung (fig. $2 b, c$ ). A partial cystectomy was performed for the cyst in the liver and because the cyst had invaded the diaphragm, it was incised and the cysts in the thorax were evacuated. Air leak test was performed by filling the thoracic cavity with saline and forceful ventilation which revealed an air leakage in the patient (fig. 2d). The broncho-biliary fistula tracts that were sutured and the diaphragm was closed. The biliary tree was irrigated with normal saline and then biliary tract involvement was investigated by giving parenterallipid solution and methylene blue from the common bile duct (fig. $2 e, f$ ). The biliary tract
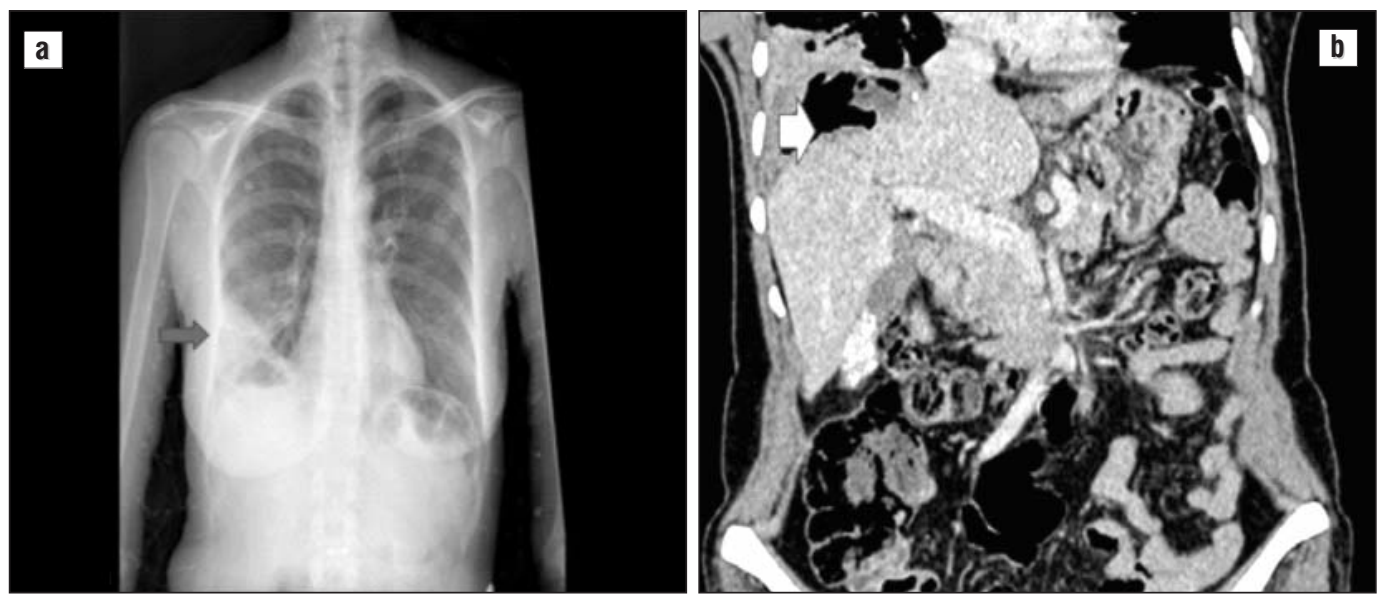

Figure 1 - This figure shows the plain chest roentgenogram and coronal computed tomography of the patient. a) shows the infiltration of the basal lobe of the right liver (arrow), b) cioronal CT slides showing the cystic cavity in the dome of the liver with air densities in side together with the subphrenic fluid collection (white arrow head) 

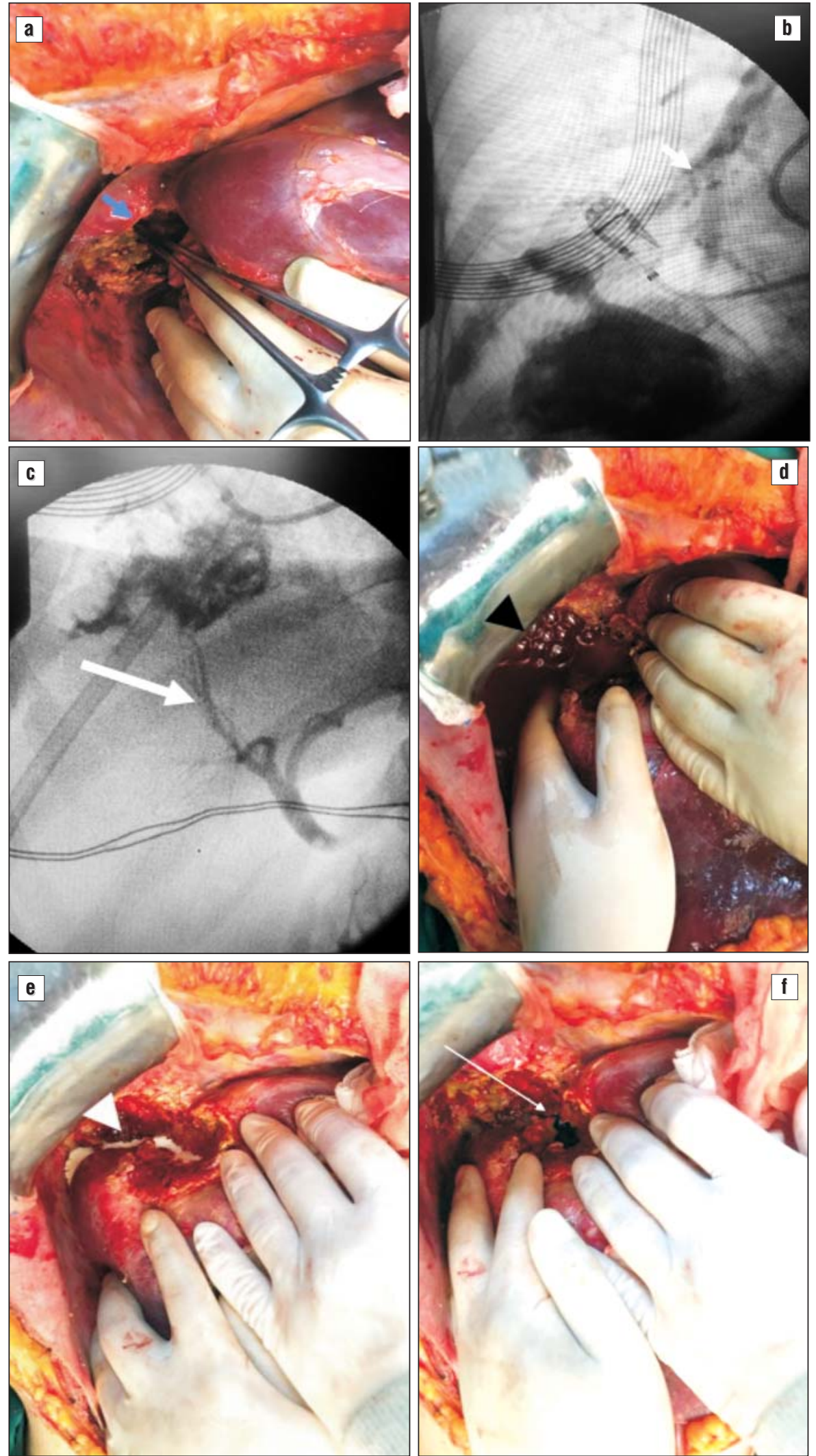

Figure 2 - Figures showing the intra-operative findings of the patient. a) Intra-operative photograph showing the cyst in the dome of the liver in close proximity with the diaphragm (Blue arrow), b) the intra-operative cholangiography showing the contrast media extravasation of the bronchi of the left lung (White arrow), c) intraoperative cholangiography showing the biliary involvement of the cyst (Thic White arrow), d) the intra-operative air leak test showing air bubbles with for ced ventilation (Black arrowhead), e) the biliary leak test performed by TPN solution (white arrow head), f) and with methylene blue (white thin arrow) 
fistula to the cyst cavity were determined and closed. After confirming that there was no bile leak, an omentopexy and falciformopexy was performed and the operation was terminated with primaryclosure of the abdomen. Albendazole $10 \mathrm{mg} / \mathrm{kg}$ was started in the early postoperative period and the patient was discharged on the postoperative $7^{\text {th }}$ day without any complication. The patient is in the postoperative $14^{\text {th }}$ month and the postoperative follow up is uneventful without any recurrences.

\section{DISCUSSION}

BBF occasionally results in pneumonia, chronic cough and dyspnea (6). Liao et al (4) made systematic review of 68 cases with BBF and evaluated the patients in two groups. In the first group there were patients with symptoms of pneumonia $(n=10,15 \%)$ and these patients had biliary stenosis and hepatocellular carcinoma as the underlying etiologies of BBF. In the second group the patients had no symptoms of pneumonia $(n=8,11.7 \%)$ and the underlying etiology was hydatid liver disease in this group (4). In the present case there was infiltration in the right basal lobe of the liver but there was no sign and symptom of pneumonia in the patient.

CT shows subphrenic collection and effusion in the thorax however it is ineffective in showing the fistula directly(7). Together with CT which shows indirect findings of thorax involvement, direct techniques such as endoscopic retrograde cholangiopancreaticography and percutaneous trans-hepatic cholangiography can be used to show the BBF tract. Magnetic resonance cholangiography and hepatobiliary scintigraphy are the two infrequently used imaging modalities, however being non-invasive modality is their main advantage (8). Magnetic resonance cholangiography is effective in showing the normal anatomy but its efficiency in showing the variations and anomalies is low (7). Suspicion of BBF and the urgent condition of the patient prompted us to proceed with surgical intervention instead of performing magnetic resonance cholangiography.

Although the diagnosis of BBF is relatively easy, the treatment of the disease is complex. If BBF developed because of treatment of the underlying disease (posttraumatic, postoperative or following percutaneous drainage etc.) endoscopic retrograde cholangiopancreaticograhy and percutaneous trans-hepatic cholangiographic drainage can be the first step in the management. In addition, in cholangitis associated with choledocholithiasis, endoscopic retrograde cholangiopancreatography can treat the underlying disease and may aid the treatment of BBF. In cases with primary pathology underlying BBF (such as advanced stage cancer) that can not be treated, endoscopic interventions may provide advantages in palliation. However, if surgical therapy can cure the underlying disease then it should be promptly applied without trying any endoscopic or radiologic procedures. Furthermore, treatment of the primary pathology as well as the BBF should be performed concomitantly in a single step. Mode of access of such a single step complex procedure is determined by the underlying disease that leads to $\operatorname{BBF}(6,9,10)$. Mode of access can be by abdominal, thoracoabdominal or occasionally thoracal route. Since the primary pathology is related with the liver, abdominal access and treatment of both clinical entities seems reasonable. Thoracoabdominal approach is extensive and may increase the complication rates and therefore should be reserved to selected cases only $(11,12)$. Biliary leaks are encountered frequently following surgery for hydatid liver disease. If the risk of biliary leak is not eliminated or reduced by intraoperative measures, postoperative prolonged hospitalization, intra-cavitary infections and adjunct interventions (endoscopic or radiologic) may be required. If the patient has $B B F$ as the presenting symptom, possibility of biliary involvement should be kept in mind and preventive measures should be taken to treat the involved bile ducts (13). The efficacy of intraoperative bile leak test during the surgery for hydatid liver disease have been shown previously (14). The present case report is the first study to emphasize the efficacy of intraoperative bile leak test in the treatment of complicated hydatid liver disease that resulted in BBF.

\section{CONCLUSIONS}

The diagnosis of BBF is a clinical entity that requires high clinical suspicion and experienced clinicians in the field. Clinical findings and radiologic evaluation are important in the diagnosis. In complicated hydatid liver disease application of intraoperative cholangiography can aid the diagnosis. Intraoperative bile leak test in hydatid liver disease reduces the risk of bile leaks and reduces the need for adjunctive postoperative interventions to treat bile fistulas.

\section{Conflict of interest}

All author declare that they have no conflict of interest. 


\section{REFERENCES}

1. Kayaalp C. Is Radical Surgery for Liver Hydatid Cyst the Right Way? World J Surg. 2016 Feb;40(2):486-7.

2. Bedioui H, Nouira K, Ayadi S, Daghfous A, Bakhtri M, Ksantini R, et al. Budd-Chiari syndrome secondary to hepatic echinococcosis. Gastroenterol Clin Biol. 2007 Sep;31(8-9 Pt 1):721-4.

3. Toumi 0, Noomen F, Salem R, Rabeh H, Jabra SB, Korbi I, et al. Intraperitoneal rupture of hydatid cysts. Eur J Trauma Emerg Surg Off Publ Eur Trauma Soc. 2017 Jun:43(3):387-91.

4. Liao G-Q, Wang H, Zhu G-Y, Zhu K-B, Lv F-X, Tai S. Management of acquired bronchobiliary fistula: A systematic literature review of 68 cases published in 30 years. World J Gastroenterol. 2011 Sep 7:17(33):3842-9.

5. Hodgdon IA, Thurston RS. Bronchobiliary fistula: a case report. J La State Med Soc Off Organ La State Med Soc. 2011;163(3):148-50.

6. Gugenheim J, Ciardullo M, Traynor 0, Bismuth H. Bronchobiliary fistulas in adults. Ann Surg. 1988 Jan;207(1):90-4.

7. Oettl C, Schima W, Metz-Schimmerl S, Függer R, Mayrhofer T, Herold CJ. Bronchobiliary fistula after hemihepatectomy: cholangiopancreaticography, computed tomography and magnetic resonance cholangiography findings. Eur J Radiol. 1999;32(3): 211-5.

8. Uramoto $\mathrm{H}$, Kawano 0 , Sugimoto $M$, Yamagata $\mathrm{H}$, Kohrogi $\mathrm{H}$. Congenital bronchobiliary fistula in a 65-year-old woman. Intern Med Tokyo Jpn. 2008;47(14):1367-70.

9. Uchikov AP, Safev GP, Stefanov CS, Markova DM. Surgical treatment of bronchobiliary fistulas due to complicated echinococcosis of the liver: case report and literature review. Folia Med (Plovdiv). 2003; 45(4):22-4.

10. Eryigit $\mathrm{H}$, Oztas $\mathrm{S}$, Urek $\mathrm{S}$, Olgac G, Kurutepe M, Kutlu CA. Management of acquired bronchobiliary fistula: 3 case reports and a literature review. J Cardiothorac Surg. 2007;2:52.

11. Tocchi A, Mazzoni G, Miccini M, Drumo A, Cassini D, Colace L, et al. Treatment of hydatid bronchobiliary fistulas: 30 years of experience. Liver Int Off J Int Assoc Study Liver. 2007;27(2):209-14.

12. Moumen M, el Fares F. [Bilio-bronchial fistula of hydatid origin. Apropos of 8 cases]. J Chir (Paris). 1991;128(4):188-92.

13. Tierris EJ, Avgeropoulos K, Kourtis K, Papaevangelou EJ. Bronchobiliary fistula due to echinococcosis of the liver. World $J$ Surg. 1977;1(1):99-104.

14. Kayaalp C, Aydin C, Olmez A, Isik S, Yilmaz S. Leakage tests reduce the frequency of biliary fistulas following hydatid liver cyst surgery. Clin Sao Paulo Braz. 2011;66(3):421-4. 